\title{
Método matemático para la sincronización de las cámaras mediante la utilización de algoritmos DDT.
}

\author{
M. GUTIÉRREZ DÁVILA , J. A. MARTÍNEZ CORRAL. \\ Departamento de Educación Física y Deportiva \\ Universidad de Granada
}

\begin{abstract}
Resumen
La utilización de los algoritmos DLT para la reconstrucción espacial de los puntos que determinan el sistema de sólidos rígidos de un deportista, requiere la filmación del gesto con dos o mas cámaras sincronizadas en el tiempo. Dicha sincronización ha constituido uno de los puntos críticos del error asociado a dichas técnicas y uno de los problemas de filmación de eventos deportivos debido al cableado que conlleva. En este trabajo se presenta un método matemático que permite dicha sincronización después de haber sido realizada la filmación. El método se basa en el error que se produce en la reconstrucción de las coordenadas $3 D$, mediante los algoritmos DLT, de un punto que está en movimiento, a partir de dos proyecciones que no coinciden en el tiempo. Dicho error se detecta mediante las diferencias entre las coordenadas digitalizadas y las proyectadas, en soporte de digitalización, del punto que ha sido reconstruido. Cuando el error es mínimo, la sincronización será la adecuada. Los resultados obtenidos han puesto de manifiesto que el método es fiables para puntos que están en movimiento, sugiriendo una metodología en el desarrollo de las técnicas fotogramétricas tridimensionales cuando se aplica a movimientos deportivos.
\end{abstract}

Palabras clave: Biomecánica.Técnica. Cinematografía

\section{Introducción}

En Biomecánica Deportiva se suelen utilizar los algoritmos de Transformación Lineal Directa $(\mathrm{DLT})^{1}$ para el análisis fotogramétrico tridimensional de los gestos deportivos, considerando al cuerpo del deportista como un sistema articulado de sólido rígidos, los cuales están definido por puntos anatómicos concretos que se desplazan en el espacio. La utilización de los algoritmos DLT para la reconstrucción espacial de los puntos que determinan el sistema de sólidos rígidos del deportista, requiere la filmación del gesto con dos o mas cámaras y la posterior obtención de las correspondientes coordenadas planas en el soporte de digitalización.

Para que la reconstrucción sea adecuada es necesario que las cámaras estén sincronizadas temporalmente, con el propósito de hacer coincidir en el tiempo las imágenes planas correspondientes a cada una de las cámaras. Dicha sincronización ha constituido uno de los puntos críticos de error asociado a las técnicas fotogramétricas tridimensionales.

Las soluciones a dicho problema han sido diversas, aunque tradicionalmente se han 
sicronizado mediante diversos soportes físicos asociados a las cámaras de video que permiten sincronizar sus campos a una frecuencia determinada o mediante eventos externos basados en luces, relojes u otros dispositivos similares, situados en el campo de visión de las cámaras y que son filmados por éstas de forma simultanea al gesto. La problemática de estos sistema, en biomecánica deportiva, es la utilización de cableado entre cámaras o la problemática que ocasiona la ubicación de los dispositivos externos en el lugar de ejecución del gesto, especialmente cuando se trata de eventos deportivos importantes.

Otras soluciones a dicho problema las han aportado Pourcelot et al $(1997)^{2}$, calculando la sincronización mediante la estimación del error medio en la reconstrucción de los puntos digitalizados, usando los algoritmos del DLT. En 1999, Yeadon \& $\mathrm{King}^{3}$, aportan un método matemático para la sincronización de cámaras de video usando, igualmente, los algoritmos DLT, calculando la raíz cuadrada de la media de los cuadrados de los valores residuales, obtenidos para los 4 planos utilizados en la matriz de reconstrucción de los puntos espaciales.

En este trabajo se propone una solución matemática que permite el reconocimiento automático, posterior a la filmación, de las imágines planas que son coincidentes en el tiempo. La base teórica que permite la sincronización de las cámaras se basa en el error que se produce en la reconstrucción de las coordenadas $3 \mathrm{D}$, mediante los algoritmos DLT, de un punto que está en movimiento, a partir de dos proyecciones que no coinciden en el tiempo (Figura 1). Cuando esto ocurre, la reconstrucción del punto en el espacio (x, $\mathrm{y}, \mathrm{z}$ ), mediante los algoritmos DLT, no coincide con la posición espacial de los puntos que han sido digitalizados $\left(\mathrm{P}_{(\mathrm{T} 0)}\right.$ y $\left.\mathrm{P}_{(\mathrm{Tn})}\right)$.

Cuando el punto $(\mathrm{x}, \mathrm{y}, \mathrm{z})$, obtenido a partir de las dos digitalizaciones mediante los algoritmos DLT, se proyecta en el soporte de digitalización, éste no coincidirá con los puntos digitalizados. Mientras que cuando el tiempo $\mathrm{T}_{\mathrm{n}}$ tiende a $\mathrm{T}_{0}$, es decir, la sincronización es correcta, deberá existir una coincidencia teórica entre los puntos digitalizados y la proyección del punto en el soporte de digitalización.

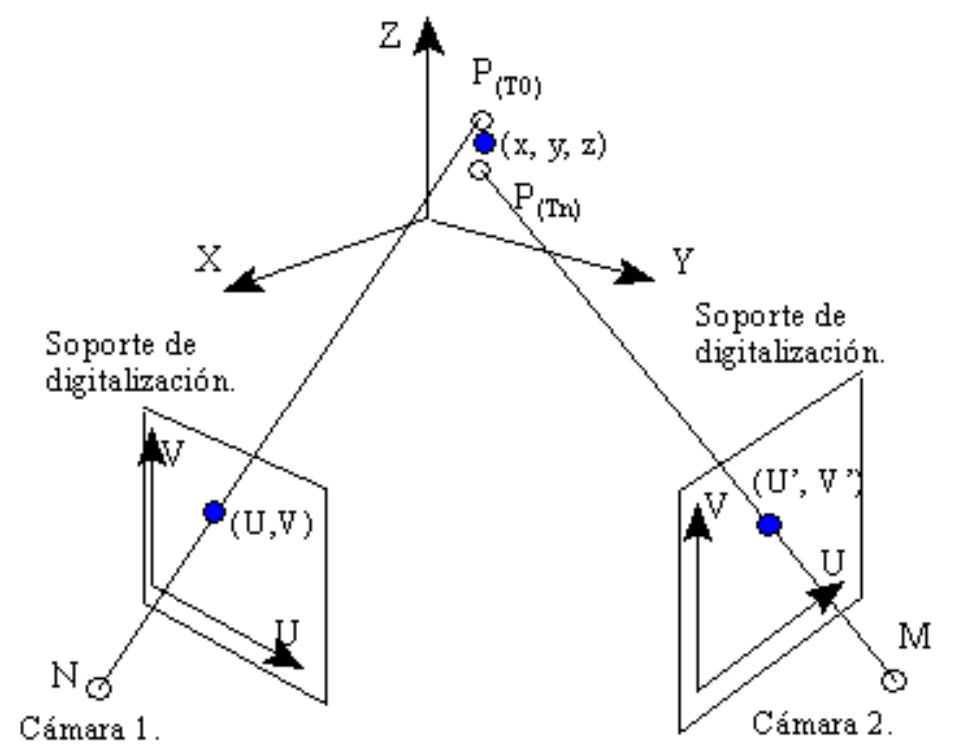

Figura 1. Representación gráfica del proceso de digitalización y reconstrucción de las coordenadas espaciales mediante el algoritmo DDT, donde (U, V) y (U', V') son las coordenadas digitalizadas de los punto $\mathrm{P}_{(\mathrm{T} 0)}$ y $\mathrm{P}_{(\mathrm{Tn})}$ respectivamente $\mathrm{y}(\mathrm{x}, \mathrm{y}, \mathrm{z})$ las coordenadas espaciales reconstruidas a partir de $(\mathrm{U}, \mathrm{V})$ y $\left(\mathrm{U}^{\prime}, \mathrm{V}^{\prime}\right)$. 
Según lo expuesto, cuando las diferencias entre el punto digitalizado y la proyección del punto reconstruido, mediante el algoritmo DLT, en el plano de imagen, tiende a 0 , el punto $\mathrm{P}_{(\mathrm{Tn})}$ tiende a
$\mathrm{P}_{(\mathrm{T} 0)}$ y se podría considerar que existe una sincronización temporal adecuada de las cámaras. El proceso matemático para llevar a cabo la teoría expuesta es la siguiente:

1.- Se parte de las constantes DLT y las coordenadas $\mathrm{x}, \mathrm{y}, \mathrm{z}$ obtenidas mediante el algoritmo DLT. Siguiendo el enunciado de la técnica de transformación directa, propuesto por el Dr. Walton (1981), la obtención de las once constantes DLT (desde A hasta L) se puede resolver mediante el siguiente sistema matricial (E-1).

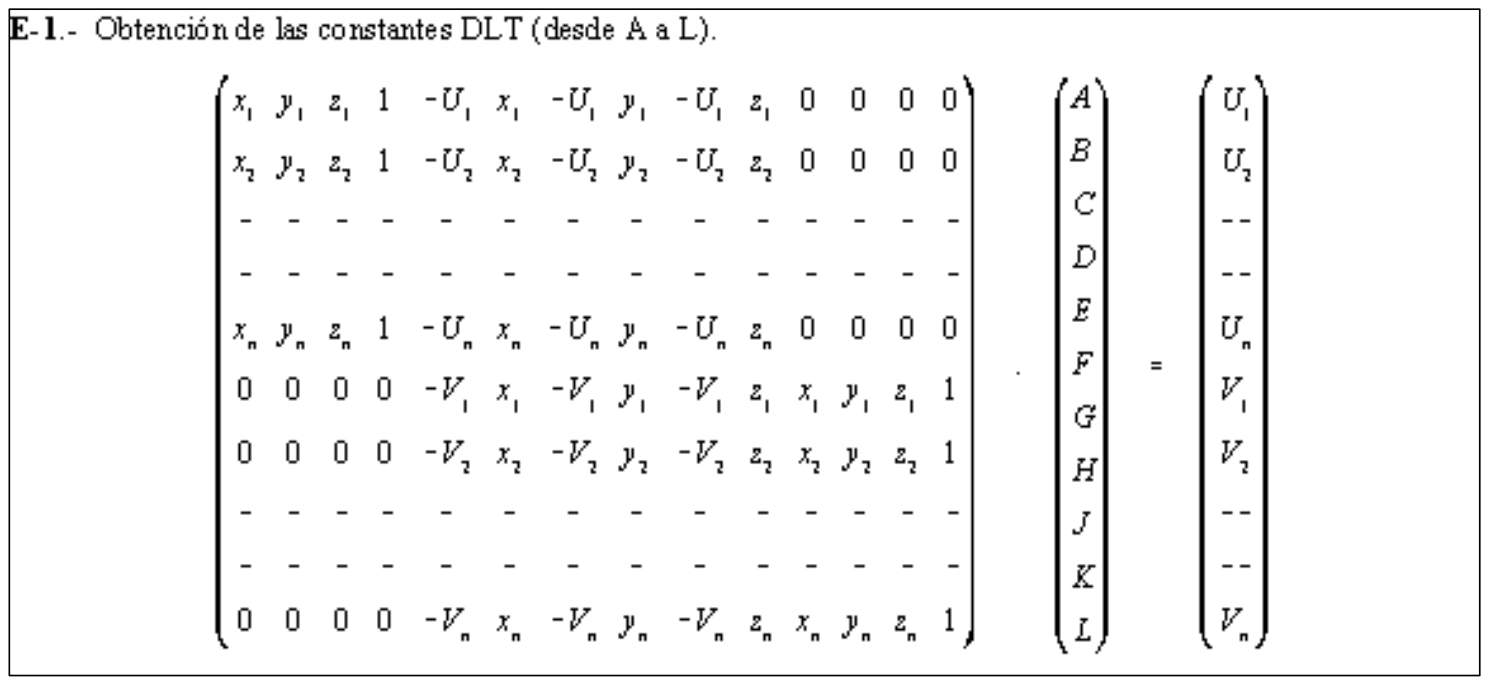

La obtención de las coordenadas espaciales (x, y, z) del punto en el espacio se resuelve mediente un sistema sobredimensionado, a partir de su digitalización en los dos planos de imagen (E-2).

E- 2.- Obtención de las coordenadas espaciales $(\mathrm{x}, \mathrm{y}, \mathrm{z})$.

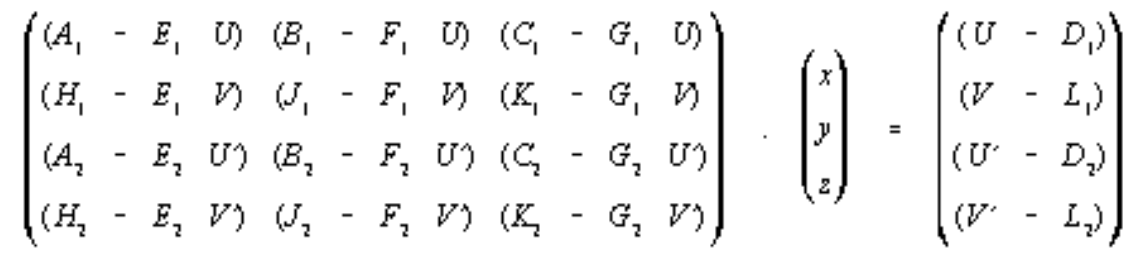

2.- Siguiendo a Kwon $(1998)^{4}$, conociendo, tanto las constantes DLT como las coordenadas del punto en el espacio y en base al desarrollo del algoritmo DLT, es posible obtener las coordenadas proyectadas del punto $(\mathrm{x}, \mathrm{y}, \mathrm{z})$ sobre cada uno de los planos de imagen, correspondiente a cada cámara (Figura 2):

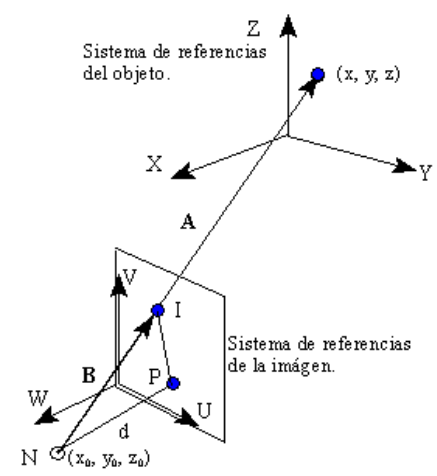

Figura 2. Representación gráfica de la relación existente entre el sistema de referencia objeto y el sistema de referencia de la imagen, donde el puto $\mathrm{N}$ es la posición de la lente de la cámara, definido por $\left(\mathrm{U}_{0}, \mathrm{~V}_{0}, \mathrm{~d}\right)$, el punto I es la proyección del punto $(\mathrm{x}, \mathrm{y}, \mathrm{z})$ en el plano de imagen, definido por $(\mathrm{U}, \mathrm{V}, 0)$ y el punto $\mathrm{P}$ es la proyección perpendicular del punto $\mathrm{N}$ en al plano de la imagen, definido por $\left(\mathrm{U}_{0}, \mathrm{~V}_{0}, 0\right)$. 
Los vectores A y B de la Figura 2 forma una línea recta con un origen común en N, coincidente con la lente de la cámara. Si estos dos vectores se definiesen en el mismo sistema de referencias, se cumpliría que $\mathbf{B}=c A$, donde $c$ es una constante de escala. Como el vector $\mathbf{A}$ está definido con respecto al sistema de referencias del objeto, es necesario expresarlo en coordenadas del sistema de referencias de la imagen. Así, el vector $\mathbf{B}$ se puede expresar a partir de una matriz de transformación. Si R es la matriz de transformación entre el sistema de referencias del objeto y el sistema de referencias de la imagen, podemos expresar la siguiente relación (E-3):

E-3 - Relación del vector $\mathbf{B}$ con la matriz de transformación $\mathrm{R}$.

$$
\begin{gathered}
B=c \cdot A_{1}=c \cdot(R \cdot A) \\
\left(\begin{array}{cc}
U & -U_{\mathrm{D}} \\
V & -V_{\mathrm{D}} \\
- & d
\end{array}\right)=c \cdot\left(\begin{array}{lll}
r_{11} & r_{12} & r_{13} \\
r_{21} & r_{22} & r_{23} \\
r_{31} & r_{32} & r_{J}
\end{array}\right) \cdot\left(\begin{array}{lll}
x & -x_{\mathrm{D}} \\
y & -y_{0} \\
z & -z_{\mathrm{D}}
\end{array}\right)
\end{gathered}
$$

Desarrollando el sistema matricial expresado en E-3 anterior y sustituyendo $c$, se obtiene la siguiente expresión (E-4):

E-4.- Desarrollo de la expresión E-3 cuando se sustituye $c$.

$$
\begin{aligned}
& U-U_{0}=-d \cdot \frac{r_{11} \cdot\left(x-x_{D}\right)+r_{12} \cdot\left(y-y_{D}\right)+r_{1 J} \cdot\left(z-z_{D}\right)}{r_{J 1} \cdot\left(x-x_{D}\right)+r_{J 2} \cdot\left(y-y_{D}\right)+r_{J} \cdot\left(z-z_{D}\right)} \\
& V-V_{\mathrm{D}}=-d \cdot \frac{r_{21} \cdot\left(x-x_{\mathrm{D}}\right)+r_{22} \cdot\left(y-y_{\mathrm{D}}\right)+r_{23} \cdot\left(z-z_{\mathrm{D}}\right)}{r_{\mathrm{Jl}} \cdot\left(x-x_{\mathrm{D}}\right)+r_{\mathrm{J}} \cdot\left(y-y_{\mathrm{D}}\right)+r_{\mathrm{J}} \cdot\left(z-z_{\mathrm{D}}\right)}
\end{aligned}
$$

Ha de tenerse en cuenta que $\mathrm{U}, \mathrm{V}, \mathrm{U}_{0}$ y $\mathrm{V}_{0}$ están en coordenadas de imagen y que éstas pueden ser diferentes de las coordenadas que se obtienen al digitalizar (en unidades del soporte donde se ha digitalizado). Teniendo en cuenta esta premisa, será necesario hacer una transformación de escala mediante las siguientes expresiones $(\mathbf{E}-\mathbf{5})$ :

E-5. Ecuaciones para la transformación de escala, donde $\lambda_{\mathrm{u}} \mathrm{y} \lambda_{\mathrm{w}}$ es el factor de escala para convertir las unidades del soporte del digitalizador en unidades de imagen.

$$
\begin{aligned}
& U-U_{D}=\lambda_{u} \cdot\left(u-u_{D}\right) \\
& V-V_{D}=\lambda_{1} \cdot\left(\nu-\nu_{D}\right)
\end{aligned}
$$

Así, las ecuaciones E-4 se pueden expresar teniendo en cuenta dicho factor (E-6):

E-6.- Desarrollo de las expresiones E-4, terie ndo en cuenta el factor escala para cada proyección $\left(\lambda_{u} \mathrm{y} \lambda_{\mathrm{v}}\right)$.

$$
\begin{aligned}
& U-U_{D}=-\frac{d}{\lambda_{u}} \cdot \frac{r_{11} \cdot\left(x-x_{\mathrm{D}}\right)+\left(r_{12} \cdot\left(y-y_{\mathrm{D}}\right)+\left(r_{13} \cdot\left(z-z_{\mathrm{D}}\right)\right.\right.}{r_{31} \cdot\left(x-x_{\mathrm{D}}\right)+\left(r_{32} \cdot\left(y-y_{\mathrm{D}}\right)+\left(r_{3} \cdot\left(z-z_{\mathrm{D}}\right)\right.\right.} \\
& V-V_{\mathrm{D}}=-\frac{d}{\lambda}, \frac{r_{21} \cdot\left(x-x_{\mathrm{D}}\right)+\left(r_{22} \cdot\left(y-y_{\mathrm{D}}\right)+\left(r_{23} \cdot\left(z-z_{\mathrm{D}}\right)\right.\right.}{r_{31} \cdot\left(x-x_{\mathrm{D}}\right)+\left(r_{32} \cdot\left(y-y_{\mathrm{D}}\right)+\left(r_{3} \cdot\left(z-z_{\mathrm{D}}\right)\right.\right.}
\end{aligned}
$$

Teniendo en cuenta la relación existente de las constantes DLT, expresadas en E-1, con respecto a la matriz de transformación y escalado y reorganizando las ecuaciones que se expresan en E-6, es posible obtener las coordenadas proyectadas sobre el soporte de digitalización del punto $(\mathrm{x}, \mathrm{y}, \mathrm{z})$ en unidades de dicho soporte (E-7): 
E-T.- Expresiones que de terminan las coordenadas proyectadas sobre el plano de imager, donde A...L, son las
constantes DLT, que indican la re lación existente entre el siste ma de referencias del objeto y el sistema de
referencias de la imagen, $\mathrm{U}_{\mathrm{T}} \mathrm{y} \mathrm{V}_{\mathrm{T}}$ son las coorde nadas proyectadas sobre el plano de imagen del punto $(\mathrm{x}, \mathrm{y}, \mathrm{z}) \mathrm{en}$
unidades del soporte de digitalización.
\[ \begin{aligned} U_{r} & =\frac{A \cdot x+B \cdot y+C \cdot z+D}{E \cdot x+F \cdot y+G \cdot z+1} \\ V_{T} & =\frac{H \cdot x+J \cdot y+K \cdot z+L}{E \cdot x+F \cdot y+G \cdot z+1}\end{aligned} \]

3.- Para un punto $\mathrm{p}$, que está en movimiento, las proyecciones en los dos soportes de digitalización de sus coordenadas $(\mathrm{x}, \mathrm{y}, \mathrm{z})$, determinadas mediante los algoritmos DLT, tendría que coincidir con sus coordenadas digitalizadas, siempre que la sincronización entre las dos imágenes sea correcta. Así, cuando la diferencia entre los tiempos de captación de las dos imágenes es igual a cero (sincronización ideal de las cámaras) y no existen otros errores asociados, se podrían expresar las siguientes relaciones (E-8):

E-8.- Situación ideal de reconstrucción de las coordenadas espaciales del punto $p$, donde $\mathrm{U}, \mathrm{V}, \mathrm{U}$ ' $\mathrm{y}^{\mathrm{V}}$ ' son las coordenadas digitalizadas, $\mathrm{U}_{T}, \mathrm{~V}_{\mathrm{T}}, \mathrm{U}^{\prime} \mathrm{r} \mathrm{y}_{\mathrm{V}}{ }_{\mathrm{r}}$, son las coordenadas proyectadas, en los dos planos de imagen, de las coordenadas espaciales del punto $p \mathrm{yd}, \mathrm{d} v, \mathrm{~d} s \cdot \mathrm{yd}$ son las dife re ncias encontradas entre las coordenadas digitalizadas y las proyectadas en cada plano.

$$
\begin{gathered}
\left(U-U_{P}\right)=d_{U}=0 ; \quad\left(V-V_{T}\right)=a_{V}=0 \\
\left(U^{\prime}-U_{P}^{\prime}\right)=a_{U^{\prime}}=0 ; \quad\left(V^{\prime}-V_{P}^{\prime}\right)=d_{V^{\prime}}=0
\end{gathered}
$$

Ya que debemos de tener en cuenta ciertos errores en el proceso de obtención de las coordenadas espaciales, unos asociados a las lentes de las cámaras, otros a la obtención de las coordenadas digitalizadas, ya sea debido al error aleatorio producido por la persona que debe realizar la digitalización o a la sensibilidad de la medida del soporte de digitalización, así como la existencia de otros errores asociados al proceso de computerización del cálculo, es necesario considerar que, aún cuando la sincronización fuese ideal, las diferencias encontradas $\left(\mathrm{d}_{\mathrm{U}}, \mathrm{d}_{\mathrm{V}}, \mathrm{d}_{\mathrm{U} \& \text { acute; }} \mathrm{y} \mathrm{d}_{\mathrm{V} \& \text { acute; }}\right)$, difícilmente podrán ser cero. Con esta situación debemos decir que, cuando las diferencias entre las coordenadas digitalizadas y las proyectadas sean mínimas, la sincronización sería la adecuada.

Posiblemente, las diferencias encontradas, no sean solo debidas a los errores expuestos, será necesario considerar, igualmente, un error asociado a la frecuencia de obtención de las imágenes, es decir, a la velocidad de las cámaras cuando éstas no están sincronizadas temporalmente mediante dispositivos internos que regulen la temporalización de los campos o del obturador, hecho que es realmente problemático conseguir. Cuando esto no ocurre, es posible que la imagen de una cámara nunca coincida temporalmente con la obtenida por la otra cámara y la sincronización se hace entre las imágenes más próximas, pudiendose producir un error de sincronización que será mayor a medida que disminuye la frecuencia de muestreo.

\section{Material y Método}

Se ha filmado, mediente dos cámaras de video a 50 imágenes por segundo, el movimiento de caída libre de una pelota de golf. Posteriormente fueron capturadas 40 imágenes para cada cámara a 640 x 480 píxeles y digitalizadas mediante un dispositivo interno que ha permitido gestionar la imagen dentro de un ordenador con una pantalla de 21" SVGA, gracias a una tarjeta capturadora de la señal de video SVGA, Jakarta, utilizándose el ratón del ordenador como marcador del punto a digitalizar y almacenando las coordenadas en unidades de píxeles ( U, V, U\&acute; y V\&acute;).
Las cámaras fueron sincronizadas mediante un dispositivo externo consistente en la emisión, a través del ordenador, de una señal TTL, la cual activaba una luz con mínima inercia de encendido (>.005 s), la cual era visible en las dos cámaras (Figura 3). Después de realizar la captura de las 40 imágenes que contenían la secuencia de movimiento de caída libre de la pelota, en las dos cámaras, se comprobó como la imagen de encendido se correspondía con la número 20 , en la cámara 1, mientras que en la cámara 2 se correspondía con la imagen 18 , es decir existía un desfase de dos fotogramas para obtener la sincronización adecuada de las cámaras. 

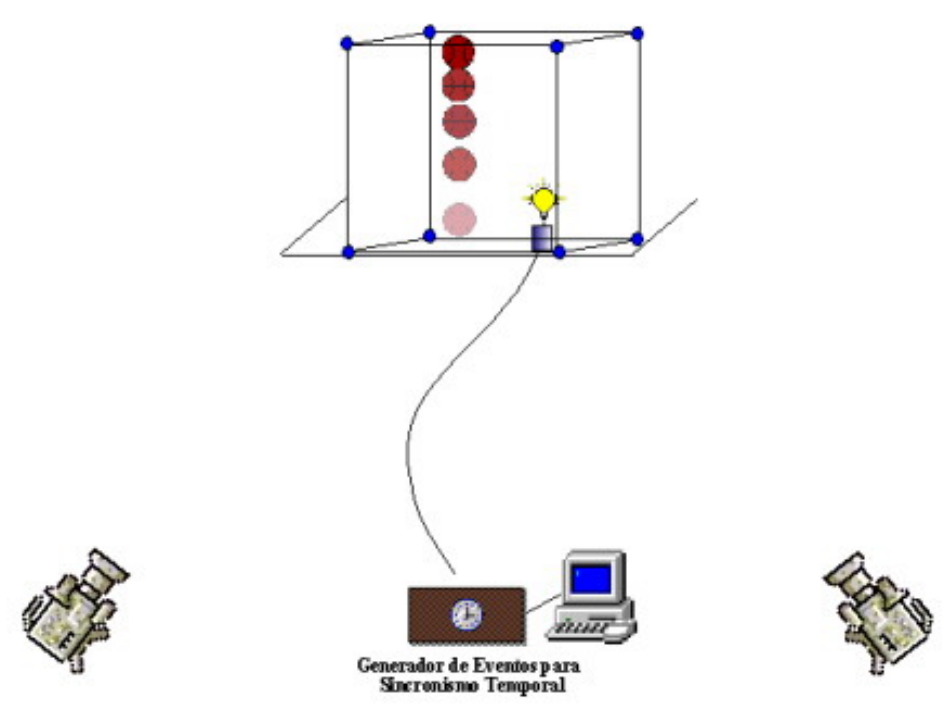

Figura 3. Filmación del movimiento de caida libre de una pelota con las cámaras sincronizadas mediante un dispositivo externo.

Nuestro propósito, ahora, es comprobar si mediante los algoritmos matemáticos propuestos, obtenemos el mismo desfase de sincronismo que el encontrado mediante el dispositivo externo de sincronización expuesto en el párrafo anterior. Para ello se ha reconstruido 21 veces el punto $(\mathrm{x}, \mathrm{y}, \mathrm{z})$ que correspondía a la pelota en movimiento, mediante los algoritmos DLT, a partir de la combinación de las coordenadas planas de la imagen 20 de la cámara 1 y las coordenadas planas de las imágenes $10,11,12,13, \ldots 30$ de la cámara 2 .

En una segunda fase, se obtuvieron las coordenadas proyectadas, del punto $(\mathrm{x}, \mathrm{y}, \mathrm{z})$, en el soporte de digitalización de la cámara $1\left(\mathrm{U}_{\mathrm{T}}, \mathrm{V}_{\mathrm{T}}\right)$, así como las diferencias encontradas con las coordenadas digitalizadas correspondientes (U, V). En la Tabla 1, se presentan las diferencias encontradas entre las coordenadas digitalizadas y las proyectadas, para las dos componentes $\left(\mathrm{d}_{\mathrm{U}}, \mathrm{d}_{\mathrm{V}}\right)$ en cada una de las combinaciones de imágenes utilizadas para la reconstrucción espacial del punto. Dichos resultados se presentan gráficamente en la Figura 4, donde se ha realizado una regresión de mínimos cuadrados a las diferencias obtenidas.

Finalmente, para comprobar si ha existido algún desfase en el sincronismo, debido a la frecuencia de muestreo utilizada, se ha repetido este mismo procedimiento matemático de sincronización, partiendo de una interpolación de las coordenadas digitalizada a $100 \mathrm{~Hz}$ utilizando para ello los algoritmos de splines elevados a la quinta potencia (Wood \& Jennings, 1979) $)^{5}$. En la Tabla 2 se presentan las diferencias entre las coordenadas obtenidas mediante la interpolación y las proyectadas.

Tabla 1.- Diferencias encontradas en la componente horizontal $\left(\mathrm{d}_{\mathrm{U}}\right)$ y vertical $\left(\mathrm{d}_{\mathrm{U}}\right)$, entre las coordenadas digitalizadas $(\mathrm{U}, \mathrm{V})$ y las proyectadas $\left(\mathrm{U}_{\mathrm{T}}, \mathrm{V}_{\mathrm{T}}\right)$, en 21 combinación de la imagen 20 de la cámara 1 con las indicadas de la cámara 2

\begin{tabular}{|c|c|c|}
\hline $\begin{array}{l}\text { Combinación } \\
\text { Imágenes. }\end{array}$ & $\begin{array}{c}\text { Diferencias } \\
\left(\mathrm{d}_{\mathrm{U}}\right) \\
(\text { píxel})\end{array}$ & $\begin{array}{c}\text { Diferencias } \\
\left(\mathrm{d}_{\mathrm{V}}\right) \\
(\text { píxel })\end{array}$ \\
\hline $20 / 10$ & 0.210767 & 16.520254 \\
\hline $20 / 11$ & 0.193258 & 14.674422 \\
\hline $20 / 12$ & 0.174959 & 12.835481 \\
\hline $20 / 13$ & 0.154875 & 10.970562 \\
\hline $20 / 14$ & 0.132027 & 9.029549 \\
\hline $20 / 15$ & 0.107403 & 7.072379 \\
\hline $20 / 16$ & 0.077096 & 4.986345 \\
\hline $20 / 17$ & 0.047186 & 2.968969 \\
\hline $20 / 18$ & 0.016657 & 0.978544 \\
\hline $20 / 19$ & 0.020794 & 1.289992 \\
\hline $20 / 20$ & 0.050268 & 3.100411 \\
\hline $20 / 21$ & 0.083117 & 5.009071 \\
\hline $20 / 22$ & 0.117688 & 6.921716 \\
\hline $20 / 23$ & 0.152358 & 8.762404 \\
\hline $20 / 24$ & 0.189366 & 10.670317 \\
\hline $20 / 25$ & 0.228618 & 12.559631 \\
\hline $20 / 26$ & 0.268184 & 14.418818 \\
\hline $20 / 27$ & 0.311307 & 16.374155 \\
\hline $20 / 28$ & 0.357825 & 18.407307 \\
\hline $20 / 29$ & 0.406688 & 20.470604 \\
\hline $20 / 30$ & 0.459966 & 22.660221 \\
\hline
\end{tabular}




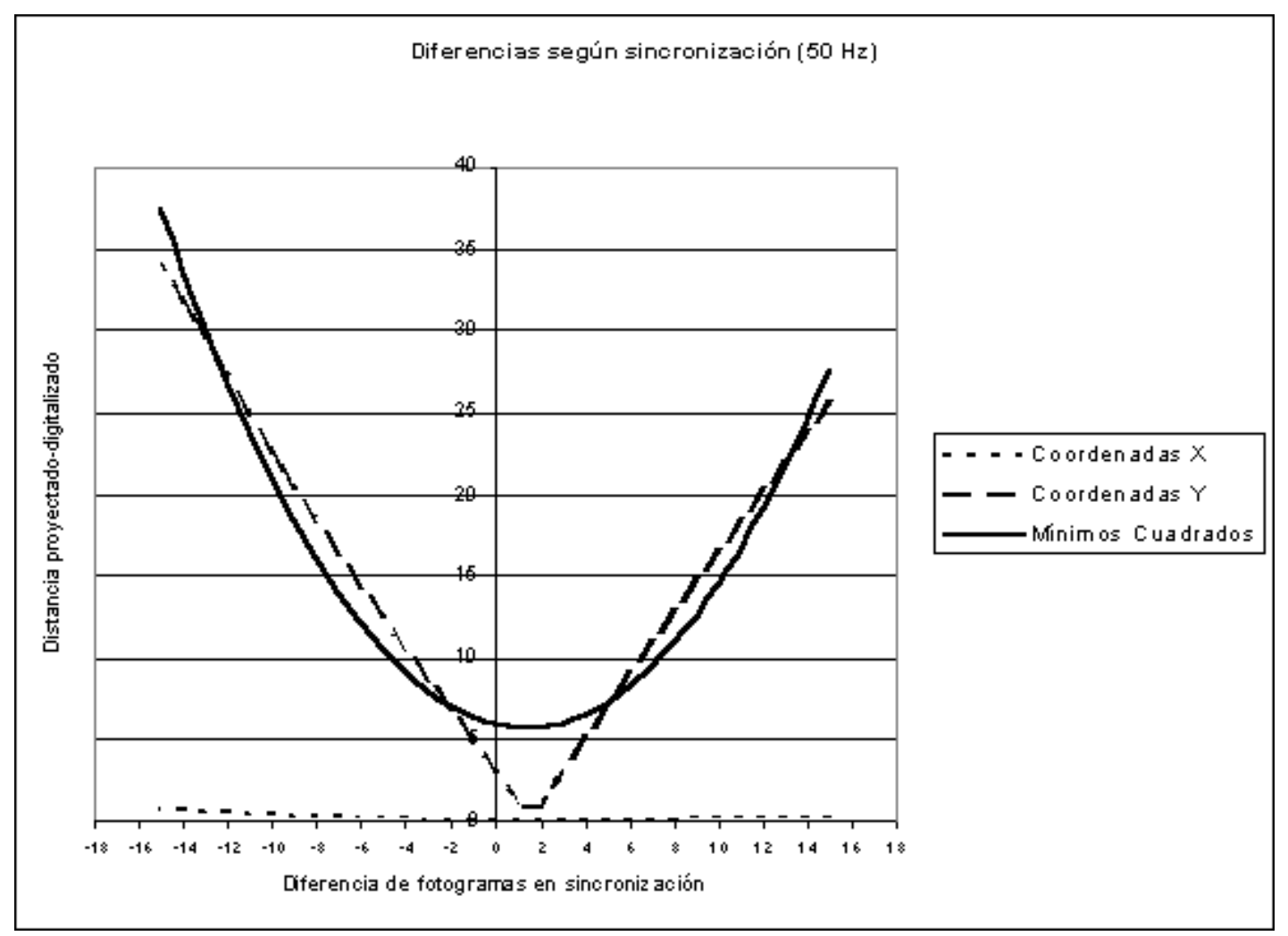

Figura 4: Representación gráfica de las diferencias encontradas entre las coordenadas digitalizadas y las proyectadas

Tabla 2.- Diferencias encontradas en la componente horizontal $\left(\mathrm{d}_{\mathrm{U}}\right)$ y vertical $\left(\mathrm{d}_{\mathrm{U}}\right)$, entre las coordenadas digitalizadas e interpoladas a $100 \mathrm{~Hz}(\mathrm{U}, \mathrm{V})$ y las proyectadas $\left(\mathrm{U}_{\mathrm{T}}, \mathrm{V}_{\mathrm{T}}\right)$, en 21 combinación de la imagen 20 de la cámara 1 con las indicadas de la cámara 2.

\begin{tabular}{|c|c|c|}
\hline $\begin{array}{l}\text { Combinación } \\
\text { Imágenes. }\end{array}$ & $\begin{array}{l}\text { Diferencias } \\
\left(\mathrm{d}_{\mathrm{U}}\right) \\
(\text { píxel }) \\
\end{array}$ & $\begin{array}{l}\text { Diferencias } \\
\left(\mathrm{d}_{\mathrm{V}}\right) \\
\text { (píxel) }\end{array}$ \\
\hline $20 / 15$ & 0,107124 & 7,106441 \\
\hline $20 / 15.5$ & 0,09205 & 6,053334 \\
\hline $20 / 16$ & 0,077195 & 5,020897 \\
\hline $20 / 16.5$ & 0,062263 & 3,995649 \\
\hline $20 / 17$ & 0,04701 & 2,968023 \\
\hline $20 / 17.5$ & 0,03128 & 1,932194 \\
\hline $20 / 18$ & 0,016111 & 0,941183 \\
\hline $20 / 18.5$ & 0,008221 & 0,480447 \\
\hline $20 / 19$ & 0,020864 & 1,315152 \\
\hline $20 / 19.5$ & 0,035488 & 2,228497 \\
\hline $20 / 20$ & 0,050806 & 3,158425 \\
\hline $20 / 20.5$ & 0,067259 & 4,127439 \\
\hline $20 / 21$ & 0,084184 & 5,10094 \\
\hline $20 / 21.5$ & 0,101597 & 6,079157 \\
\hline $20 / 22$ & 0,119026 & 7,039062 \\
\hline $20 / 22.5$ & 0,136642 & 7,988474 \\
\hline $20 / 23$ & 0,154647 & 8,936613 \\
\hline $20 / 23.5$ & 0,173122 & 9,887989 \\
\hline $20 / 24$ & 0,192128 & 10,847189 \\
\hline $20 / 24.5$ & 0,21151 & 11,807754 \\
\hline $20 / 35$ & 0,23117 & 12,763912 \\
\hline
\end{tabular}

\section{Discusión}

Se comprueba como, en la Tabla 1, tanto las diferencias encontradas en la componente horizontal $\left(\mathrm{d}_{\mathrm{U}}\right)$ como en la vertical $\left(\mathrm{d}_{\mathrm{V}}\right)$ son mínimas en la combinación (20/18), no llegando a superar la sensibilidad de la medida del soporte de digitalización (1pixel) y coincidente con la sincronización encontrada mediante el dispositivo externo de sincronización. A medida que se incrementa el tiempo entre las dos imágenes, utilizadas para reconstruir el punto en el espacio mediante los algoritmos DLT, las diferencias encontradas $\left(\mathrm{d}_{\mathrm{U}}, \mathrm{d}_{\mathrm{V}}\right)$ se incrementan, siendo más marcadas en la componente vertical, debido a que su desplazamiento, en dicha componente, es mayor al tratarse de un movimiento de caída libre.

Según los resultados expuestos, debemos de considerar que cuanto mayor es el desplazamiento del punto, mayor será la sensibilidad de la medida $\mathrm{y}$, consecuentemente, la fiabilidad del método. Mediante este método no sería posible sincronizar las cámaras seleccionando un punto que no se desplaza en el espacio, es necesario seleccionar previamente el punto que tiene mayor desplazamiento en el plano donde se realizará la proyección. 
Aplicando, a estas coordenadas planas, el método propuesto por Yeadon \& $\mathrm{King}^{3}$, los resultados obtenidos son coincidentes (Figura 4), siendo mayor la sensibilidad de la medida obtenida mediante el método que se propone en este trabajo, además de simplificarse el proceso de cálculo y poder obtener el error de digitalización mediante la mínima diferencia después de calcular el sincronismo adecuado.

Cuando se repite este mismo procedimiento matemático de sincronización, partiendo de una interpolación de las coordenadas digitalizada a 100 imágenes por segundo, se comprueba como las diferencias mínimas encontradas se reducen y el sincronismo adecuado no sería 20/18, sino el 20 / 18.5. Se comprueba como, en este caso, se estaba produciendo un cierto error de sincronismo debido a la frecuencia de muestreo $(50 \mathrm{~Hz})$ que se reduce cuando previamente se interpola a $100 \mathrm{~Hz}$. En este sentido, debemos sospechar que para movimientos deportivos rápidos, la sincronización de las cámaras debe realizarse a frecuencias de muestreo por encima de $100 \mathrm{~Hz}$ y en movimientos especialmente rápidos como golpeos o movimientos balísticos, la frecuencia debe ser mayor.

\section{Conclusiones.}

El método de la mínima diferencia entre las coordenadas digitalizadas y las proyectadas en el soporte de digitalización, desarrollado en este trabajo, es fiable para la sincronización de las cámaras cuado se pretende obtener un punto en el espacio que está en movimiento. Para obtener una sincronización adecuada de las cámaras, cuando se utilizan las técnicas fotogramétricas tridimensionales en biomecánica deportiva, se propone la siguiente metodología:

a) Realizar la digitalización en un soporte de máxima sensibilidad (incrementar la resolución de la imagen cuando se trata de soportes informáticos).

b) Detectar el punto del sistema que posee mayor desplazamiento en el soporte de digitalización donde se va a proyectar la imagen espacial. c) Interpolar las coordenadas planas procedentes de las digitalizaciones de las dos cámaras sin realizar suavizado de dichos datos.

d) Obtener las coordenadas espaciales a partir de distintas combinaciones de imágenes planas. Para reducir el número de combinaciones posibles y simplificar el proceso, introducir un evento común visible en las dos imágenes, como un apoyo o cualquier contacto del deportista con un sólido externo.

e) Proyectar las imágenes espaciales obtenidas en el soporte de digitalización, calculando así nuevas coordenadas planas en el soporte donde se había detectado mayor desplazamiento del punto y obtener la combinación de imágenes planas donde se ha determinado la mínima diferencia entre las coordenadas digitalizadas y las proyectadas.

f) Calcular las coordenadas espaciales de todos los puntos que definen el sistema de sólidos rígidos del deportista, en toda la secuencia, utilizando la combinación de imágenes determinada en el punto anterior.

\section{Bibliografía}

1. Abdel-Aziz, Y.I. \& Karara, H.M. Direct linear transformation fron comparator coordinates into object space coordinates in close-range photogrammetry". Proc. ASP/UI Symp. Close-Range Photogrammetry, Urbana, Illinois. Falls Church, V.A: American Society of Photogrammetry, 1971; 118

2. Pourcelot, P., Audigiè, F., Degueurce, C. Denoix, J.M. \& Geiger, D. A numerical method to synchronise home video cameras using the D.L.T. method. Medical and Biological Engineering and Computing. 1997; 35, 2: 715.

3. Yeadon, M.R. \& King, M.A. A method for synchronising digitised video data. Journal of Biomechanics. 1999; 32: 983-986.

4. Kwon. DLT Method (1998). http://www.cs.bsu.edu/ ykwon/kwon3d/dlt/dlt.htm

5. Wood, G.A. \& Jennings. On the use of spline functions for data smoothing". Journal of Biomechanic. 1979; 12: 477-479. 